Indications for Medical Treatment--(1) Biliary dysfunction without radiological evidence of organic disorder. (2) A mild degree of chronic cholecystitis in which there is no evidence of the presence of gallstones, particularly in young people. (3) Large stones in older people with little clinical evidence of dyspepsia due to their presence. (Newly developed gastro-intestinal symptoms in such patients are probably due to some other cause.) (4) Single cholesterol stones discovered by accident. (5) Multiple pigment stones. (Operation should be deferred until investigation for haemolysis has been undertaken.)

\section{Treatment of Biliary Colic}

For severe gallstone colic, despite theoretical arguments against its use, nothing can replace full doses of morphine by injection. It is true that morphine acts only by deadening the pain; it will not abort the attack. The theoretical argument against the use of morphine is somewhat specious, and depends upon the fact that in some individuals morphine increases an already existing plain-muscle spasm. In gallstone colic the gall-bladder is trying to expel a gallstone by powerful contractions, and will give up the effort only when it tires, or is successful, or ruptures (a very rare consequence). In the milder attacks of biliary colic associated with hypertonia and not due to gallstones, the argument against using morphine is valid, for in them the pain is presumably due to hypertonia caused by muscle spasm, which morphine may indeed encourage. For these cases antispasmodics ought to be tried, especially when such attacks are frequent. Inhalation of amyl nitrite may give relief, and so may atropine, $1 / 75 \mathrm{gr}$. $(0.87 \mathrm{mg}$.). Antihistamine injections are unlikely to succeed. If antispasmodics are of no avail then pethidine should be preferred to morphine.

\section{Acute Cholecystitis}

Acute cholecystitis may occur in quite young peopleeven children-without any pre-existing disease in the gallbladder. It is probably a primary bacterial infection in these circumstances, due to Bact. coli or, more rarely in this country, arising as a sequel to typhoid. The condition usually resolves with medical treatment, and the question of surgery does not arise.

However, in by far the greater number of cases the acute attack is as an exacerbation of a pre-existing chronic cholecystitis, and gallstones are usually present. There is thus an antecedent history of flatulent dyspepsia to aid in the diagnosis. The immediate predisposing cause of the attack is obstruction at the outlet of the gall-bladder, usually by a stone, but possibly by a plug of mucus or oedema of the mucosa. The severity of the illness will depend upon the degree of obstruction and the virulence of the superimposed infection.

The signs and symptoms of acute cholecystitis are too well known to need recapitulation here. Emphasis may, however, be laid upon the common association with it of a transient and not very deep jaundice due to infection ascending into the intrahepatic ducts and not to a stone in the common bile duct, to hyperaesthesia on the right side posteriorly over the lower ribs, to pain in the right shoulder or over the right scapula, to retrosternal pain, or to an associated disordered action of the heart in older people.

Whether the treatment of acute cholecystitis should be medical or surgical cannot be decided from the depths of an armchair, nor from a study of statistical tables. Each case ought to be decided on its clinical merits, with a bias against operation. Latterly there has been a mass movement-one of those curious and inexplicable changes of surgical opinion -away from conservation towards operation. The new eagerness to operate upon the acutely inflamed gall-bladder -an operation which may if performed early be deceptively easy-seems to coincide with a sharp increase in the incidence of that major surgical catastrophe, injury to the common bile duct. Priestly avers that strictures of the bile duct due to this cause have increased threefold in recent years.
What is to be feared in acute cholecystitis is sloughing of the gall-bladder wall, with perforation into the general peritoneal cavity, an event which rarely follows acute primary cholecystitis in young people. Judgment of the probability of this complication should be based on the severity of the initial pain, and on the rapidity of progress and the degree of the local and general inflammatory changes. Operative difficulty, and therefore mortality, rises steeply as the hours pass, and if a decision to operate be made it should be acted upon without delay. After the second day the gall-bladder region is likely to be occupied by an inflammatory mass of forbidding extent, and added emphasis should be laid on the desirability of conservative treatment. If the surgeon feels impelled to operate at this stage, and especially if the patient is ageing or obese, he would be well advised to pocket his technical pride and limit the procedure to drainage of the gall-bladder. If a stone is firmly impacted in Hartmann's pouch, lying deep in the wound, and its removal from this plane a difficult task, it would be best to leave it there in spite of the probability that the drainage wound will not heal. Simple drainage is likely to ensure survival, and the patient will doubtless weather the second operation if it be postponed for some weeks after all traces of inflammatory activity have disappeared.

In the conservative treatment of acute cholecystitis streptomycin and chloramphenicol are the antibiotics of choice.

\section{Torsion}

Torsion of the gall-bladder, a rare condition, is most likely to affect the elderly visceroptotic subject, whose gallbladder is unusually mobile, and, though containing stones, is free from gross disease. The onset is dramatic, for the pain is unheralded and agonizing, but is instantaneously relieved when the gall-bladder ruptures. Immediate cholecystectomy must be undertaken-an operation which is made easy by the build of the patient and the mobility of the gall-bladder.

\section{Cancer}

Of cancer of the gall-bladder there is little to write, for if it is clinically recognizable it is probably incurable; fortunately it is a comparatively rare condition. The evidence that calculus cholecystitis predisposes to cancer of the gall-bladder is overwhelming, and this is a powerful argument in favour of cholecystectomy in the treatment of the former condition.

Next Refresher Course Article.- “ Rheumatoid Arthritis," by Dr. W. S. C. Copeman.

\section{A. SYRINGE SERVICE FOR A DEPARTMENT OF ANAESTHETICS}

BY

T. H. S. BURNS, B.M., B.Ch., D.A.

Research Fellow, Department of Anaesthetics, Guy's Hospital; Late Senior Resident Anaesthetist, Guy's Hospital

In February, 1948, a syringe service was introduced for our department of anaesthetics. This has recently been modified in the light of three years' experience. The service works well, and none of the component parts is difficult to obtain.

The aim of the syringe service is to present syringes and needles to the anaesthetist in containers which: (1) may be easily and reliably sterilized ; (2) involve the minimum of handling, thus reducing the possibility of infecting a sterile syringe while assembling it and drawing up the drug; (3) enable the contents to be easily identified ; (4) involve a minimum of labour to the staff ; (5) have a reasonably long life ; (6) will stand safely on 
a trolley, without risk of their rolling off ; 7 ) are made of easily obtainable materials ; and (8) hold the syringe firmly, and so reduce the risk of damage to the syringe during transport and sterilization.

\section{The Apparatus}

It was decided that syringes should be all-glass, since metal-and-glass syringes, however well washed, are apt to retain small particles of blood which may cause homologous serum jaundice any time within six months of their being used for intravenous injection (M.R.C., 1945). The new type of metal-and-glass syringe in which an alloy is used with a similar coefficient of expansion to that of glass was investigated. The advantage of such a syringe is its metal nozzle, which is much less fragile than one made of glass. The plunger is all-glass, for ease of cleaning, and the assembled syringe may be sterilized in the hot-air oven or the autoclave. Unfortunately, even in these syringes the cement between the metal and the glass tended to disintegrate on repeated sterilization, and the cavity which was left provided a lodging-place for blood that it was almost impossible to clean thoroughly.

Our all-glass syringes have peripheral nozzles, with a Luer mount, and conform to British Standard 1263, 1946. Plungers and barrels have identifying numbers, and each plunger is always assembled with its own barrel. Syringes are of 5,10 , and $20 \mathrm{ml}$.

The needles are made of stainless steel, and are in three sizes:-size 1: mixing-16 s.w.G. by 3 in. $(7.6 \mathrm{~cm}$.); size 2 : injecting-25 s.w.G. by $\frac{7}{8}$ in. $(2.2 \mathrm{~cm}$.) ; and size 3 : injecting -21 s.w.G. by $1 \frac{3}{8}$ in. $(3.5 \mathrm{~cm}$.). Sizes 2 and 3 have a 45-degrees straight bevel.

The syringe containers consist essentially of glass tubes 11 in. $\left(28 \mathrm{~cm}\right.$.) long and $1 \frac{3}{8}$ in. $(3.5 \mathrm{~cm}$.) wide. Glass tubing of this bore is easily obtainable, being used in the sterilization of gum-elastic catheters. Each end is closed by a rubber bung, also easily obtainable and designed to fit that particular tubing. After repeated autoclaving the rubber tends to shrink, and it has been found desirable to insert a tapered cork inside the bung. This cork can be forced in far enough to make the rubber a tight fit in the glass tube. Both the cork and the rubber bung have holes drilled in them to allow steam to enter the container during autoclaving. A small amount of cotton-wool is placed between the cork and the bung so that the contents remain sterile when withdrawn from the autoclave.

One end of the tube is made into a support for the barrel of the syringe by bending a piece of 20-gauge aluminium or similar metal 1 by 9 in. $(2.5$ by $23 \mathrm{~cm}$.) into the shape shown in the illustration. A strip of adhesive orthopaedic

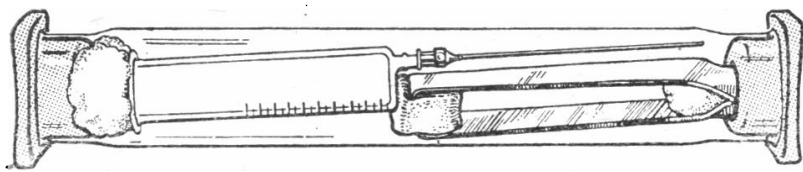

felt $\frac{3}{8}$ by $3 \frac{1}{4}$ by $\frac{1}{4}$ in. ( 10 by 83 by $6 \mathrm{~mm}$.) is placed on the central end of the metal and folded under. The opposite end of the longer limb is curved to fit between the cork and the rubber bung. This, together with the felt at the other end, holds the support firm in the container. The other end of the glass tube is closed by another rubber bung with cork and cotton-wool inserted.

The needle containers consist of test-tubes 3 by $\frac{1}{2}$ in. (7.6 by $1.3 \mathrm{~cm}$.) with coloured cotton-wool plugs. The point of the needle is protected by slipping over it a piece of 5-mm.bore glass tubing $1 \frac{5}{8}$ in. $(4.1 \mathrm{~cm}$.) long.

\section{Cleaning and Packing}

A bowl of cold water is provided in each anaesthetic room, and it is the responsibility of the anaesthetist to see that syringes are rinsed and then dismantled and placed in the water immediately after use. This may be done by a nurse, student, or orderly, who should rinse out the needle at the same time. The importance of rinsing the syringes in cold water immediately after use cannot be emphasized too strongly. Only in this way is it possible to avoid blood clotting in the apparatus.

Syringes are later washed in warm soapy water and, after rinsing, dried with a fluffless cloth. Lubrication consists in dipping the tip of the plunger in liquid paraffin and rubbing the paraffin well into the ground surface with the finger. Care is taken to use no more liquid paraffin than is necessary. The piston is inserted into the barrel and worked backwards and forwards to ensure proper lubrication. After attaching the wide-bore mixing-needle the syringe is inserted into its glass tube. Removal of the syringe is facilitated by attaching the mixing-needle in such a way that the broad part of its base lies parallel to the metal support in the glass container. There is then no risk of the base of the needle jamming between the metal support and the side of the container. The syringe is inserted into the container so that syringe and mixing-needle lie parallel with the lower surface of the glass, the base of the syringe pressing against the felt covering of the metal support. The cotton-wool packing can then fill in any gap between the head of the plunger and the opposite surface of the glass, as well as filling in the interval between plunger head and rubber bung. A "tail" of the cotton-wool packing is left between the lip of the container and the rubber bung. This enables the cotton-wool to be removed without touching the inside of the container. The syringe is thus prevented from rattling in its container and the mixing-needle lies free in air, no strain being put on the nozzle of the syringe. The standard 11 -in. $(28-\mathrm{cm}$.) tube thus serves for the three different sizes of syringe.

Needles are washed with warm water from a small syringe and the mount is cleaned with cotton-wool on the end of a swab-stick. They are rinsed in cold water, then in alcohol, and allowed to dry. A wire stylet, lubricated with a minimal amount of liquid paraffin, is passed through the bore to ensure patency. The stylet is removed and the points of the needles are examined. Those which are unsatisfactory are replaced by new or sharpened needles. The unsatisfactory ones are sent to be sharpened. Apart from this daily check, all needles are changed once every week. The sharp needles, without their stylets, are assembled in test-tubes. They are plugged with red cotton-wool if they are 25 s.W.G. and green cotton-wool if they are 21 s.w.G. These needles are packed in drums and sterilized with the syringes.

Sterilization.-Syringes and needles, in their respective containers, are autoclaved at $120^{\circ}$ C. $(15-20 \mathrm{lb} .-1-1.4 \mathrm{~kg}$ per $\mathrm{cm}^{2}$ - pressure) for 20 minutes. The rubber bungs will not stand up to the $170^{\circ} \mathrm{C}$. necessary for sterilization in a hot-air oven. The effectiveness of this method of sterilization was checked by inoculating three assembled syringes with Staph. aureus and three with Bacillus mycoides (a nonpathogenic spore-bearing rod). Three cleaned and assembled syringes were used as controls. After sterilization no growth in broth was obtainable from any syringe.

\section{Method of Use}

A selection of sterile syringes and a drum of needles are placed on each anaesthetist's table. The anaesthetist chooses the size of syringe he requires and, after opening his thiopentone or other ampoules, removes the rubber bung and cotton-wool packing from the glass container. The syringe is allowed to slide into the hand and the thiopentone can then be mixed directly, as the mixing-needle is already attached to the syringe. When the mixture is finally drawn up, the wide-bore needle is discarded and the needle for injection selected according to the anaesthetist's choice and the colour of the cotton-wool plugging the test-tube. The wool is removed without touching the lip of the tube, and the nozzle of the syringe is applied to the tube. The tube and syringe are inverted together, when the needle mount slips on to the nozzle. After securing the needle tightly the syringe is ready for use. 
The flanges of some makes of 20-ml. syringes are too large to fit into the glass tubing used for the containers. We are at present experimenting with glass tubing $1 \frac{5}{8}$ in. $(4.1 \mathrm{~cm}$.) in diameter which would fit all these syringes. However, suitable rubber bungs are not easily obtainable to fit this size, and at present we find it more convenient to confine our purchases to a make of $20-\mathrm{ml}$. syringe which will fit our existing tubes. "Accoson" is one make which is suitable.

The complete packages, as well as the individual items, may be obtained from Messrs. Down Bros.

I am grateful to Mr. A. D. Marston, the director of our department, for his continual encouragement and help throughout the evolution of this service. The hospital anaesthetists and the nursing staff have been more than sympathetic in the way in which they have co-operated in maintaining the service.

\section{REPERENCE}

Medical Research Council (1945). Sterilization, Use and Care of Syringes.

\section{Nova et Vetera}

\section{A GREAT FRENCH SURGEON}

The Apologie and Treatise of Ambroise Paré. Edited, with an introduction, by Geoffrey Keynes, M.D., F.R.C.S. F.R.C.O.G. (Pp. 227; 15s.) London: Falcon Educational Books. 1951.

Ambroise Paré (1510-90) was, beyond question, one of the great surgeons of all time. His figure dominated the surgical and deeply affected the medical practice of his century. None of his works is easily accessible in English. The seventeenth-century translations of them are costly and the various modern selections have long been out of print. For this fine selection Mr. Keynes has used the translation of Thomas Johnson (1634), who was probably the same as the editor of the second edition of Gerarde's Herball (1633).

The manly figure of Paré can never lose its attractions. At once feariess and discreet both in act and word, witty, wise, and expert, a master of his native French but of no other language, humane, alert, and of complete integrity, Paré was as complete a man as can be found. Mr. Keynes's account of him fails only by its brevity, for we could wish some tabulated and chronological account of his career and more copious notes than are here provided. But the book is illuminated in another way, for, in addition to the numerous text figures, it is adorned by four perfectly delightful portraits of Paré, displaying his magnificent head passing from vigorous middle life to healthy age.

Paré's contribution to surgery is usually summarized by the mention of his three "discoveries"- the harmfulness of treating gunshot wounds with boiling oil, the use of the ligature in amputation, and podalic version in obstetricsbut in reality his contribution was far greater. He was, in fact, " by virtue of his personality and independent mind, the emancipator of surgery from the dead hand of dogma. There was no comparable practitioner, during his time, and his influence was felt in every part of Europe." His collected Works is a monument of skill and humanity unsurpassed in the history of surgery. His books are packed with exciting incidents and case-histories, told with a picturesqueness well reflected by his translator. No bad sample is the famous passage in which he relates his conversion from the violent current method of treating gunshot wounds. Therefore let Paré speak for himself :

"In the yeare of our Lord 1536, Francis the French King sent a puissant Army beyond the Alpes, under the leading of Annas of Mommorancie, high Constable of France, that he might releeve Turin.... In this conflict there were many wounded on both sides, cheefely with bullets. I will tell the truth; I was not very expert at that time in matters of Chirurgery; neither was I used to dresse wounds made by Gunshot. Now I had read that wounds made by Gunshot were poisoned by reason of the Gunpouder ; wherefore for their cure, it was expedient to cauterize them with oyle scalding hot. Before I would runne a hazard, I determined to see whether the Chirurgions, who went with me in the army, used any other manner of dressing to these wounds. I saw that all filled as full as they could, the wounds made by Gunshot with pledgets dipped in this scalding Oyle, at the first dressings ; which encouraged me to doe the like to those, who came to be dressed of me. It chanced on a time, that by reason of the multitude that were hurt, I wanted this Oyle. Now because there were some few left to be dressed, I was forced, that I might not leave them undrest, to apply a digestive made of the yolke of an egge, oyle of Roses, and Turpentine. I could not sleepe all that night, for I was troubled in minde, and the dressing of the precedent day (which I judged unfit) troubled my thoughts; and I feared that I should finde them at the point of death by the poyson of the wound, whom I had not dressed with the scalding oyle. Therefore I rose early and, beyound expectation, I found such as I had dressed with a digestive onely, free from vehemencie of paine, to have had good rest, and that their wounds were not inflamed; but the others were feaverish, tormented with much paine, and the parts about their wounds were swolne. When I had many times tryed this in divers others, I thought thus much, that neither I nor any other should ever cauterize any wounded with Gun-shot."

Charles Singer.

\section{Preparations and Appliances}

\section{A SYRINGE GAUGE FOR INSULIN}

Dr. J. C. Pease and Mr. R. Salt, of Oxford, write : The diabetic patient with failing eyesight often finds it difficult to measure the prescribed dose of insulin. To overcome this hazard we have designed an apparatus which can be pre-set by the physician and clipped on to an Everett 1-ml. syringe. The patient fills the syringe with insulin, and while the needle is in the insulin bottle he attaches the apparatus to the syringe and pushes the plunger in as far as the stop (Fig. 2 shows this action completed). The needle is then withdrawn from the bottle, the apparatus removed, and the injection given in the usual way. The apparatus is made in two parts: $A$ and $C$ (Fig. 1). Part A clips over the length of the syringe. It is drilled with a series of holes which are threaded to take the knurled locking bolt, $B$. The holes are placed to allow an adjustment in $0.1-\mathrm{ml}$

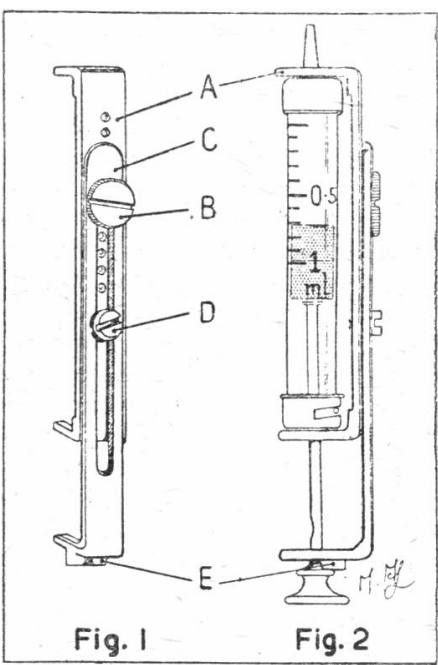

stages up to $1 \mathrm{ml}$. Part $\mathrm{C}$ is slotted and drilled with one hole. Bolt $\mathrm{D}$ passes through the slot. This bolt cannot be completely removed; it thus prevents the two parts from being separated, but when it is slackened it allows the slotted part $\mathrm{C}$ to slide over part A. Movement is prevented by passing bolt $B$ through the hole and screwing it into a threaded hole in part A. Both bolts are made to take a sixpennypiece to facilitate adjustments. Fig. 2 shows the apparatus in position on the syringe with the plunger pushed down to stop E. This apparatus is available through Longworth Scientific Instrument Co., Ltd., Abingdon, Berks. 